\title{
28. Behandlung der Dystrophia musculorum progressiva durch kombinierte Injektion von Adrenalin und Pilokarpin.
}

\author{
Von Ken KurÉ und Shigeo OKINaKa. \\ Medizinische Klinik (Abt. von Prof. K. Kuré) der Kaiserlichen \\ Universität zu Tokyo. \\ (Rec. Jan. 30, 1930. Comm. by K. Miura, M.I.A., Feb. 12, 1930.)
}

Dass die Dystrophia musculorum progressiva auf einer Störung der autonomen Muskelinnervation beruht, wurde uon unserer Klinik auf Grund der experimentellen und pathologisch-anatomischen Untersuchungsergebnisse erwiesen. Schon 1920, als Kuré eine solche Entstehungsweise der Dystrophie vermutete, hat er damals unternommen, sie durch Adrenalininjektionen zu behandeln. Dabei konnte er an 3 unter 6 Dystrophikern dadurch deutliche Besserung der Muskelkraft erzielen. 7 Jahre später hat Tsuji an 2 Dystrophikern, die ihren Vorderarm noch genügend bewegen konnten, und an 4 Kranken, deren Halssympathicus exstirpiert war, den Einfluss des Adrenalins und Pilokarpins auf die Ermüdbarkeit der Vorderarm- und Handmuskeln untersucht. Adrenalin verzögerte hierbei immer den Eintritt der Ermüdung an dystrophischen und sympathicotomierten Muskel, wobei jedoch der dystrophische Muskel stärker auf Pilokarpin anzusprechen schien. Da es nach den Untersuchungen unserer Klinik als festgestellt gilt, dass bei der Dystrophie meist auch die parasympathische Muskelinnervation affiziert ist, so ist es wahrscheinlich, dass die Empfindlichkeit gegen Pilokarpin infolge der Affektion der parasympathischen Innervation gesteigert ist, gerade wie der glatte Muskel durch die Enervierung gegen autonome Gifte überempfindlich wird. Diese Untersuchung von Tsuji hat gleichzeitig gezeigt, dass der sympathicotomerte Muskel noch nach mehreren Jahren auf Adrenalin abnorm stark reagiert.

Nun erhebt sich die Frage, ob die wiederholten Injektionen von Adrenalin und Pilokarpin auf den dystrophischen Muskel heilenden Einfluss haben?

Diesbezüglich ist das Resultat von Harada's Experiment bemerkenswert. Er konnte nemlich durch wiederholte Injektionen des Tetanustoxins (kleiner als 1/100 der Letaldosis) in den M. semitendinosus des Hundes Hypertrophie des Muskels hervorrufen. Wenn man nun 
in den dystrophischen Muskel, welcher durch die Affektion der autonomen Innervation verändert ist und doch noch den Angriffspunkt für autonome Gifte beibehält, Adrenalin und Pilokarpin wiederholt injiziert und den Mangel der zentralen autonomen Innervation durch periphere Giftwirkung ersetzt, so ist es möglich, dass das Fortschreiten der Dystrophie dadurch gehemmt, ja sogar dadurch eine Restitutio ad integrum herbeigeführt wird. Unter dieser Voraussetzung haben wir versucht, den Dystrophiker durch wiederholte kombinierte Injektionen von Adrenalin und Pilokarpin zu behandeln.

Adrenalin wurde in $0.1 \%$ iger Lösung in der Menge von $0.2-0.3$ $\mathrm{ccm}$, Pilokarpin in 1\% iger Lösung zu $0.1-0.2 \mathrm{~cm}$ subkutan injiziert. Nach der Injektion klagten die Kranken selten über ein gewisses Mattigkeitsgefühl. Die Injektion wurde täglich oder jeden zweiten Tag wiederholt, wenigstens 50-60 mal, an einem Falle 120 mal mit Unterbrechungen.

Behandelt wurden 12 Patienten. 2-3 Stunden nach der Injektion bemerkt man deutliche Kräftesteigerung an dystrophischen Muskeln. Diese Wirkung dauert gewöhnlich 12 Stunden. Nach verschieden häufig wiederholten Injektionen bemerkt man dauernde Kraftzunahme. Dies kam in einem Falle schon nach 15 Injektionen, in einem anderen aber erst nach 30 Injektionen zu stande. Besserung der Muskelkräfte, die nach 50-60 Injektionen auftrat, bestand mehrere Monate lang nach dem Sistieren der Injektionen. In zwei Fällen unter 12 Dystrophikern erhielten wir einen eklatanten Erfolg. Die Patientin, die vor der Injektion nur mit Krücke kaum 100 Meter gehen konnte, war nach 40 Injektionen im Stande krückenlos 4-5 Kilometer weit zu kommen. Ihr Körpergewicht nahm während der Behandlung zu. Bei ihr wurde der Gang nach zweimonatlicher Unterbrechung der Injektion nur wenig schlechter. Der andere Dystrophiker, der seine Arme kaum höher als bis zum Horizontalen heben konnte, vermochte nach 16 Injektionen seine Arme $45^{\circ}$ über die Horizontale zu heben. Nach 50 Injektionen konnte er seine Arme in der gehobenen Stellung halten. Die Muskulatur war so deutlich hypertrophiert, dass es bei blossem Anblick leicht erkennbar war. Der Umfang des Oberschenkels $(20 \mathrm{~cm}$ oberhalb der Mitte der Patella) betrug vor der Behandlung rechts $31.0 \mathrm{~cm}$, links $35.0 \mathrm{~cm}$, am Ende der Behandlung rechts 33.5, links $37.0 \mathrm{~cm}$. Er konnte nach seiner Entlassung seinen Beruf als Erdarbeiter ohne Störung erfüllen. 3 Monate sind schon nach seiner Entlassung verflossen, ohne dass man über eine Verschlimmerung seines Krankheitszustandes etwas zu hören. Andere 6 Kranke zeigten eine gewisse 
Besserung, die durch Untersuchung mit dem Ergographen deutlich feststellbar war. Verschiedene Verrichtunge und Körperhaltungen, die vor der Behandlung kaum möglich waren, wurden nach der Behandlung leichter ausgeführt, ohne dass aber vollständige Heilung eintrat. Die letzten 3 Fälle, die hochgradige Dystrophie zeigten und mit spinaler progressiver Muskelatrophie kompliziert waren, zeigten keinen deutlichen Erfolg dieser Behandlung; doch war das Fortschreiten der Atrophie, die bis zur letzten Zeit rasch vor sich ging, während der Injektionen einstweilen sistiert. Im ganzen kann man wohl sagen, dass der Erfolg der Behandlung desto besser war, in je früherem und komplikationslosem Stadium die Krankheit zur Behandlung kam. Besonders wichtig scheint uns, dass die Übung der betreffenden Muskeln während der Injektionen für eine Restitutio ad integrum notwendig ist, wozu noch gewisse Kraft der dystrophischen Muskel erhalten bleiben muss. Die Besserung stellt sich gewöhnlich während der ersten 50 Injektionen ein; kann darnach kein genügender Erfolg erzielt werden, so ist die Aussicht auf Erfolg sehr zweifelhaft. Was die Nebenwirkung der Behandlung betrifft, so tritt meist bald nach der Injektion etwas Herzklopfen auf. Nach 50-120 maliger Injektion konnte man weder Hypertrophie des Herzens noch dauernde Blutdrucksteigerung nachweisen. Man beobachtet aber gelegentlich, dass andere gesunde Muskeln z.B. der M. gastrocnemius u.s.w. während dieser Behandlung stark hypertrophieren.

Vor kurzem hat Stscherbak über Adrenalinbehandlung der Dystrophia musculorum progressiva publiziert. Er glaubt, dass die Dystrophie durch Adrenalininjektion in gewissen Fällen vollständig heilen kann. Wir haben in günstigen Fällen eine auffallende Besserung und fast vollständige Funktionswierstellung gesehen; trotzdem glauben wir nicht, dass die Dystrophie durch diese Behandlung vollständig und dauernd geheilt werden kann. Da die Besserung und Hypertrophie der dystrophischen Muskeln durch Ersetzen des mangelnden zentralen Impulses durch peripherische Giftwirkung angestrebt wird, so ist es möglich, dass die Dystrophie nach Unterbrechung der Adrenalin- und Pilokarpininjektion wieder weiter forstchreitet. Da aber das Fortschreiten der Dystrophie, wie bekannt, sehr langsam vor sich geht, so kann man vielleicht durch wiederholte Kuren dasselbe bis $\mathrm{zu}$ einem gewissen Grade hemmen. In diesem Sinne ist die genannte Behandlung empfehlenswert, weil zur Zeit keine andere bessere Heilmethode bekannt ist. 